\title{
Body Mass Index and Chronic Health Conditions as Predictors of Exercise Fear-Avoidance Beliefs
}

\author{
Brooks C. Wingo ${ }^{1}$, Jamy D. Ard ${ }^{2}$, Renee Desmond ${ }^{3}$, Retta Evans ${ }^{4}$, Jane Roy ${ }^{4}$ \\ and Monica Baskin ${ }^{3}$ \\ ${ }^{1}$ Department of Occupational Therapy, University of Alabama at Birmingham, Alabama, USA \\ ${ }^{2}$ Department of Epidemiology and Prevention, Wake Forest School of Medicine, North Carolina, USA \\ ${ }^{3}$ Division of Preventive Medicine, University of Alabama at Birmingham, Alabama, USA \\ ${ }^{4}$ Department of Human Studies, University of Alabama at Birmingham, Alabama, USA
}

Correspondence should be addressed to: Brooks C. Wingo; bcwingo@uab.edu

Received 6 August 2013; Accepted 4 September 2013; Published 19 November 2013

Academic Editor: Achim Elfering

Copyright (C 2013 Brooks C. Wingo, Jamy D. Ard, Renee Desmond, Retta Evans, Jane Roy and Monica Baskin. Distributed under Creative Commons CC-BY 3.0

\begin{abstract}
Obese adults often experience heightened physical responses to exercise, and some may interpret this response as a sign that they are in danger of harm or injury. This fear of harm, or fearavoidance beliefs (FAB), leads some individuals to avoid exercise, leading to increased sedentary behavior and further difficulty maintaining weight. Evidence indicates that body mass index (BMI) is predictive of $F A B$, but no research has considered the impact of medical conditions on FAB in obese adults. The purpose of this study was to assess the impact of cardiorespiratory and musculoskeletal conditions on FAB. Participants $(n=155)$ completed the Exercise Fear Avoidance Scale (EFAS), a checklist of medical conditions, and the Pain Disability Index. We calculated differences in EFAS scores between groups with and without each condition using t-tests. We used linear regression to calculate the amount of variance in EFAS scores accounted for by BMI, age, pain and condition. Individuals reporting a cardiorespiratory or musculoskeletal condition had significantly higher EFAS scores than those who did not ( 7.4 points, $\mathrm{P}<.001 ; 4.4$ points, $\mathrm{P}=.001$, respectively). BMI, pain and age explained significant portions of the variance in EFAS scores $(P=.003 ; P<.001 ; P=.001$, respectively). When added to the regression equations, no condition significantly added to the variance in EFAS scores. While those with cardiorespiratory and musculoskeletal conditions may have higher FAB than those without, BMI appears to contribute to this fear even in individuals without these conditions. Practitioners should consider FAB when discussing exercise recommendations with obese individuals regardless of cardiorespiratory or musculoskeletal history.
\end{abstract}

Keywords: Fear-Avoidance Beliefs, Obesity, Physical Activity, Barriers.

Cite this Article as: Brooks C. Wingo, Jamy D. Ard, Renee Desmond, Retta Evans, Jane Roy and Monica Baskin (2013), "Body Mass Index and Chronic Health Conditions as Predictors of Exercise Fear-Avoidance Beliefs," Journal of Research in Obesity, Vol. 2013 (2013), Article ID 793181, DOI: 10.5171/2013.793181 


\section{Introduction}

Physical activity is a key component of behavioral interventions for obesity, but exercise can be a particularly difficult behavior for individuals who are overweight or obese to initiate. Sedentary individuals who begin to increase physical activity will often experience physical discomfort as a result of increased movements, and these discomforts can be worse in overweight or obese individuals. Discomfort may be due to increased daily pain associated with obesity, as well as heightened physical response to exertion experienced by obese individuals compared to normal weight adults (Ekkekakis \& Lind, 2006; Nevitt \& Lane,1999; Tukker, Visscher \& Picavet, 2009). This increased response may be in the form of joint pain, which can be made worse by the additional force exerted on joints as a result of higher body weight in obese individuals (Nevitt \& Lane, 1999; Tukker, et al., 2009). Increased response may also take the form of cardiorespiratory discomfort. Overweight and obese individuals have been found to experience increased symptoms of cardiovascular exertion including increased perceived exertion and oxygen consumption compared to sedentary individuals of normal weight when performing an equal amount of work. This may result in a higher rate of perceived exertion and decreased enjoyment of activity (Ekkekakis \& Lind, 2006).

Some overweight or obese adults may interpret the discomfort experienced during exercise as a sign that they are in danger of harm or injury. In turn, these individuals may refrain from exercise that might aid in the achievement of a healthier weight. The fearavoidance model of chronic pain proposes that if an individual perceives pain as a sign of harm, he or she may assume the cause of the pain is dangerous and believe that there is an underlying serious illness or injury. This catastrophic thinking often leads the individual to ruminate on the pain and the assumed cause of the pain, leaving the person with a hyperawareness of any painful sensation. If left unaddressed, this cycle of thinking can lead to avoidance of activity (Lethem, Slade, Troup, \& Bentley, 1983; Vlaeyen, Kole-Snijders, \& Rotteveel, 1995).

Evidence suggests there is a direct relationship between fear of exerciseinduced pain, or exercise fear-avoidance beliefs, and increased body mass index (BMI; Wingo et al, 2013). In previous research, higher BMI was significantly associated with higher scores on a measure of exercise fearavoidance. Additionally, exercise fearavoidance scores were negatively associated with weekly minutes of physical activity, such that higher fear-avoidance scores predicted lower levels of physical activity (Wingo et al., 2013). One potential explanation for the relationship between BMI and exercise fear-avoidance may be the existence of other conditions such as osteoarthritis or coronary artery disease (CAD) that often occur with increased BMI. Conditions such as osteoarthritis may increase the amount of pain/discomfort experienced during exercise, leading an individual to believe the pain is a sign of harm. Additionally, qualitative research suggests that the presence of cardiorespiratory risk factors and conditions such as hypertension or heart disease may lead an individual to believe their discomfort is a sign of potential danger (Wingo et al., 2011). The purpose of this study was to determine if co-morbid medical conditions commonly reported by obese adults contribute to the previously established relationship between BMI and exercise fearavoidance beliefs. We hypothesized that the presence of a cardiorespiratory or musculoskeletal condition would significantly contribute to the variance in fear-avoidance beliefs explained by BMI alone.

\section{Methods}

Participants were 20-65 year old adults recruited from a University-based clinical weight loss program and from the general 
community. The primary inclusion criterion for this study was a BMI of $18.5-60 \mathrm{~kg} / \mathrm{m}^{2}$. An upper limit was set for BMI because of the potential for an extreme BMI to skew results as outliers. Individuals who were under instructions to avoid physical activity or limit exercise due to a medical condition were also excluded. These instructions may influence participants' perception of the safety of exercise and therefore skew responses by elevating FAB. This study was approved by the University Institutional Review Board and informed consent was obtained from each participant.

\section{Measures}

Exercise Fear Avoidance Scale (EFAS). The EFAS was designed to measure fear of physical response to exercise among overweight and obese adults in three domains: weight-specific fear, cardiorespiratory fear and musculoskeletal fear (Wingo et al., 2013). The total scale consists of 16 items. Items are one-sentence statements (e.g. I need to lose weight before I can exercise safely; Pain during exercise means I am injuring myself), and respondents are asked to rate their agreement/disagreement with each statement on a 5-point Likert scale: (1) Strongly Disagree, (2) Disagree, (3) Neither Agree nor Disagree, (4) Agree, and (5) Strongly Agree. Scores on the total scale range from 16- 80. Subscale scores range from 6-30 on the weight-specific fears subscale and 5-25 on both the cardiorespiratory fears and musculoskeletal fear subscales. Higher scores indicate higher fear-avoidance beliefs.

Validation studies of the EFAS demonstrated sound psychometric properties. Internal consistency of the EFAS was $\alpha=.86$. Scores on the EFAS were significantly associated with BMI such that those individuals with a higher BMI indicated higher fear scores. EFAS scores have also been associated with physical activity level such that higher fear scores accurately predicted lower levels of self-reported physical activity (Wingo et al., 2013).

Medical Diagnoses. Participants were given a list of common medical diagnoses and asked "Have you ever been told that you have any of the following medical conditions?" Conditions were grouped into three categories based on the type of condition. Cardiorespiratory conditions included high blood pressure, coronary artery disease, heart disease other than coronary artery disease, heart attack, stroke, mitral valve prolapse, heart murmur, pulmonary hypertension, COPD, asthma, diabetes, high cholesterol, and obstructive sleep apnea. Musculoskeletal conditions included fibromyalgia, arthritis, joint pain other than arthritis, plantar fasciitis, osteoporosis, herniated disk, and degenerative joint disease. Other conditions included acid reflux or GERD, Crohn's disease, chronic sinusitis, polycystic ovarian syndrome (PCOS), migraines, and chronic fatigue syndrome.

Many of the diagnoses were those often associated with obesity (e.g. hypertension, diabetes), however others were also included (e.g. migraines). The checklist was completed after the EFAS was completed to avoid biasing participants' responses to the EFAS. Self-report of medical conditions was used because fear of harm is based on patient perception. That is, if an individual believes he has a particular condition he will respond to perceived risk of harm associated with this condition even if he has not truly been given this diagnosis.

Body Mass Index. Patients were weighed in light clothing without shoes, using a Tanita digital scale (Model \#BWB500A). Height was measured using a wall-mounted stadiometer. BMI was calculated using the formula kilograms $/$ meters $^{2}$ (Garrow \& Webster, 1985). 
Pain-related disability. Pain-related disability was measured using the Pain Disability Index (PDI; Pollard, 1984). This scale asked participants to measure the effect of pain on seven aspects of life, including family and home responsibilities, recreation, social activities, occupation, sexual behavior, and life-support activities. Participants were asked to rate the effect of pain on a scale of 0 to 10 , with 0 indicating no disability at all, and 10 indicating total disability. The scale was scored by adding the responses to the seven categories. Scores range from 0-70, with higher scores indicating greater disability.

\section{Data Analysis}

We calculated differences in EFAS scores between individuals who reported having each medical condition and those who did not using independent-samples t-tests. For those conditions where there was a significant difference between those who reported the condition and those who did not, we used linear regression to calculate the amount of variance in EFAS scores accounted for by the condition. The initial model included BMI, PDI and age. The condition was then entered to assess the significance of change to the total amount of variance explained by the new model. All analyses were conducted using SPSS software version 18 (SPSS Inc, Chicago, IL).

\section{Results}

A total of 155 participants completed the study. Table 1 shows the summary statistics of the participants. This was a middle-aged sample of mostly female participants. There were no significant differences on total EFAS scores between males and females $(P=.24)$. There were also no significant differences in total EFAS scores based race or education level $(P=.11, P=.10$, respectively). The mean (SD) BMI of the sample was $35.17(8.86)$ $\mathrm{kg} / \mathrm{m}^{2}$. Eighty-three percent of participants reported having at least one of the conditions on the medical condition checklist.

Table 1: Participant Characteristics

\begin{tabular}{|l|c|c|c|}
\hline $\mathrm{n}=155$ & Range & Mean (SD) & $\mathrm{n} \%$ \\
\hline Age (years) & $20-65$ & $45.1(11.7)$ & \\
\hline Gender & & & $73.5 \%$ \\
Female & & & $26.5 \%$ \\
Male & & & \\
\hline Ethnicity & & & $27.7 \%$ \\
African American & & & $68.4 \%$ \\
Caucasian & & $35.17(8.86)$ & $1.9 \%$ \\
Hispanic & $18.5-55.6$ & & $14.2 \%$ \\
\hline BMI $\left(\mathrm{kg} / \mathrm{m}^{2}\right)$ & & $16.1 \%$ \\
$\quad$ Normal weight $\left(18.5-24.9 \mathrm{~kg} / \mathrm{m}^{2}\right)$ & & & $23.2 \%$ \\
$\quad$ Overweight $\left(25.0-29.9 \mathrm{~kg} / \mathrm{m}^{2}\right)$ & & & $16.8 \%$ \\
$\quad$ Class I Obesity $\left(30.0-34.9 \mathrm{~kg} / \mathrm{m}^{2}\right)$ & & & $29.7 \%$ \\
$\quad$ Class II Obesity $\left(35.0-39.9 \mathrm{~kg} / \mathrm{m}^{2}\right)$ & & & \\
$\quad$ Class III Obesity $\left(>40.0 \mathrm{~kg} / \mathrm{m}^{2}\right)$ & & $10.14(14.09)$ & \\
\hline Pain Disability Index & $0-56$ & & \\
\hline
\end{tabular}

\section{Differences in Fear Scores Based on Medical Conditions}

Of 28 conditions listed on the checklist, only celiac was not reported by any participant.
Several other conditions were reported by only a small number of participants. For purposes of this analysis, conditions were only analyzed if 5 or more participants reported having the condition. Participants 
reporting at least one cardiorespiratory condition or risk factor scored 7.35 points higher on the total EFAS scores on average than those participants reporting that they did not have any of the cardiorespiratory conditions or risk factors $(P<.001)$. Individuals who reported at least one cardiorespiratory condition or risk factors had significantly higher BMIs than those who did not report any cardiorespiratory conditions or risk factors $(P<.001$; table 2$)$. They were also significantly older $(P<.001)$ and reported more pain disability $(P<.001)$ than those without a cardiorespiratory condition or risk factor. Participants reporting hypertension scored 4.73 points higher on the EFAS $(P=.001)$ on average that those without hypertension. Those reporting a heart murmur scored 4.15 points higher on average than those without $(P=.02)$. Participants with pulmonary hypertension scored 7.94 points higher on average $(P$ $=.04$ ). Participants with high cholesterol or obstructive sleep apnea also had significantly higher fear-avoidance scores than those who did not (4.36, $P=.002$ and 5.02, $P=.001$, respectively). For each of these conditions, differences on the weight-specific subscale were greatest, followed by differences on the cardiorespiratory fear subscale, and the musculoskeletal fear subscales (table 3).

Table 2: BMI, Pain Disability and Age Based on Medical Conditions

\begin{tabular}{|c|c|c|c|c|c|c|c|}
\hline Condition & $\mathrm{n}(\%)$ & \multicolumn{2}{|c|}{$\begin{array}{c}\text { BMI }\left(\mathrm{kg} / \mathrm{m}^{2}\right) \\
\text { Mean }(\mathrm{SD})\end{array}$} & \multicolumn{2}{|c|}{$\begin{array}{c}\text { PDI } \\
\text { Mean (SD) }\end{array}$} & \multicolumn{2}{|c|}{$\begin{array}{l}\text { Age (years) } \\
\text { Mean (SD) }\end{array}$} \\
\hline \multicolumn{8}{|c|}{ Condition Reported } \\
\hline & & Yes & No & Yes & No & Yes & No \\
\hline $\begin{array}{l}\text { Any cardiorespiratory } \\
\text { condition }\end{array}$ & $\begin{array}{c}109 \\
(70.3)\end{array}$ & $\begin{array}{c}36.99 \\
(8.46)^{* *}\end{array}$ & $\begin{array}{l}29.76 \\
(7.83)\end{array}$ & $\begin{array}{c}12.63 \\
(14.96)^{* *}\end{array}$ & $2.74(7.25)$ & $\begin{array}{c}48.34 \\
(10.20)^{* *}\end{array}$ & $\begin{array}{c}35.49 \\
(10.69) \\
\end{array}$ \\
\hline Hypertension & $\begin{array}{c}64 \\
(41.3)\end{array}$ & $\begin{array}{c}37.91 \\
(7.94)^{* *}\end{array}$ & $\begin{array}{l}33.25 \\
(9.00) \\
\end{array}$ & $\begin{array}{c}13.03 \\
(13.54)^{*}\end{array}$ & $\begin{array}{c}8.11 \\
(14.19)\end{array}$ & $\begin{array}{c}51.28 \\
(8.71)^{* *}\end{array}$ & $\begin{array}{c}40.77 \\
(11.64)\end{array}$ \\
\hline Mitral valve prolapse & $14(9)$ & $\begin{array}{l}34.87 \\
(5.45)\end{array}$ & $\begin{array}{l}35.20 \\
(9.14)\end{array}$ & $\begin{array}{c}10.86 \\
(15.57)\end{array}$ & $\begin{array}{c}10.07 \\
(13.99)\end{array}$ & $\begin{array}{c}50.14 \\
(6.68)^{*}\end{array}$ & $\begin{array}{c}44.61 \\
(12.00)\end{array}$ \\
\hline Heart murmur & $10(6.5)$ & $\begin{array}{l}38.57 \\
(7.92) \\
\end{array}$ & $\begin{array}{l}34.94 \\
(8.89) \\
\end{array}$ & $\begin{array}{c}19.30 \\
(17.60)^{*}\end{array}$ & $\begin{array}{c}9.51 \\
(13.67) \\
\end{array}$ & $\begin{array}{l}49.60 \\
(9.22) \\
\end{array}$ & $\begin{array}{l}44.80 \\
(11.83\end{array}$ \\
\hline Pulmonary hypertension & $5(3.2)$ & $\begin{array}{c}38.46 \\
(11.56) \\
\end{array}$ & $\begin{array}{l}35.06 \\
(8.78) \\
\end{array}$ & $\begin{array}{c}29.4 \\
(20.73)^{* *}\end{array}$ & $\begin{array}{c}9.50 \\
(13.45) \\
\end{array}$ & $\begin{array}{c}52.00 \\
(4.74)^{*}\end{array}$ & $\begin{array}{c}44.88 \\
(11.81) \\
\end{array}$ \\
\hline Asthma & $\begin{array}{c}24 \\
(15.5)\end{array}$ & $\begin{array}{c}34.83 \\
(10.41) \\
\end{array}$ & $\begin{array}{l}35.24 \\
(8.59) \\
\end{array}$ & $\begin{array}{c}10.04 \\
(13.67) \\
\end{array}$ & $\begin{array}{c}10.16 \\
(14.24) \\
\end{array}$ & $\begin{array}{c}45.05 \\
(10.90) \\
\end{array}$ & $\begin{array}{c}45.12 \\
(11.89) \\
\end{array}$ \\
\hline Diabetes & $\begin{array}{c}23 \\
(14.8) \\
\end{array}$ & $\begin{array}{c}38.61 \\
(7.25)^{*}\end{array}$ & $\begin{array}{l}34.57 \\
(9.00) \\
\end{array}$ & $\begin{array}{c}15.87 \\
(13.21)^{*}\end{array}$ & $\begin{array}{c}9.14 \\
(14.05) \\
\end{array}$ & $\begin{array}{c}47.87 \\
(11.68) \\
\end{array}$ & $\begin{array}{c}44.63 \\
(11.69) \\
\end{array}$ \\
\hline High Cholesterol & $\begin{array}{c}60 \\
(38.7) \\
\end{array}$ & $\begin{array}{c}36.23 \\
(7.32) \\
\end{array}$ & $\begin{array}{l}34.51 \\
(9.68) \\
\end{array}$ & $\begin{array}{c}12.58 \\
(14.36) \\
\end{array}$ & $\begin{array}{c}8.60 \\
(13.77) \\
\end{array}$ & $\begin{array}{c}50.25 \\
(8.97)^{* *}\end{array}$ & $\begin{array}{c}41.86 \\
(12.11) \\
\end{array}$ \\
\hline Sleep Apnea & $\begin{array}{c}43 \\
(27.7)\end{array}$ & $\begin{array}{c}39.23 \\
(8.40)^{* *}\end{array}$ & $\begin{array}{l}33.61 \\
(8.56)\end{array}$ & $\begin{array}{c}15.60 \\
(17.79)^{* *}\end{array}$ & $\begin{array}{c}8.04 \\
(12.85)\end{array}$ & $\begin{array}{c}49.63 \\
(9.33)^{* *}\end{array}$ & $\begin{array}{c}43.38 \\
(12.10)\end{array}$ \\
\hline Any musculoskeletal condition & $\begin{array}{c}70 \\
(45.2)\end{array}$ & $\begin{array}{c}37.09 \\
(7.90)^{*}\end{array}$ & $\begin{array}{l}33.59 \\
(9.32)\end{array}$ & $\begin{array}{c}15.93 \\
(16.78)^{* *}\end{array}$ & $5.38(9.05)$ & $\begin{array}{c}50.47 \\
(9.11)^{* *}\end{array}$ & $\begin{array}{c}40.69 \\
(11.82)\end{array}$ \\
\hline Arthritis & $\begin{array}{c}42 \\
(27.1)\end{array}$ & $\begin{array}{c}38.64 \\
(7.97)^{* *}\end{array}$ & $\begin{array}{l}33.88 \\
(8.85)\end{array}$ & $\begin{array}{c}20.86 \\
(17.55)^{* *}\end{array}$ & $\begin{array}{c}6.16 \\
(10.06)\end{array}$ & $\begin{array}{c}52.55 \\
(8.37)^{* *}\end{array}$ & $\begin{array}{c}42.35 \\
(11.60)\end{array}$ \\
\hline Joint pain other than arthritis & 24 & 39.77 & 34.33 & 20.13 & 8.31 & 49.50 & 44.31 \\
\hline
\end{tabular}




\begin{tabular}{|c|c|c|c|c|c|c|c|}
\hline & $(15.5)$ & $(7.53)^{* *}$ & $(8.85)$ & $(18.24)^{* *}$ & $(12.43)$ & $(8.24)^{*}$ & (12.09) \\
\hline Plantar fasciitis & $\begin{array}{c}29 \\
(18.7)\end{array}$ & $\begin{array}{l}37.95 \\
(7.22)\end{array}$ & $\begin{array}{c}34.53 \\
(9.10)\end{array}$ & $\begin{array}{c}11.21 \\
(13.78)\end{array}$ & $\begin{array}{c}9.90 \\
(14.20)\end{array}$ & $\begin{array}{l}48.31 \\
(9.10)\end{array}$ & $\begin{array}{c}44.37 \\
(12.15)\end{array}$ \\
\hline Herniated Disc & $17(11)$ & $\begin{array}{l}36.60 \\
(8.81) \\
\end{array}$ & $\begin{array}{r}34.10 \\
(8.88) \\
\end{array}$ & $\begin{array}{c}17.06 \\
(17.22)\end{array}$ & $\begin{array}{c}9.29 \\
(13.49)\end{array}$ & $\begin{array}{c}48.18 \\
(11.58)\end{array}$ & $\begin{array}{c}44.73 \\
(11.71)\end{array}$ \\
\hline Degenerative Joint Disease & $13(8.4)$ & $\begin{array}{r}38.19 \\
(9.31) \\
\end{array}$ & $\begin{array}{l}34.90 \\
(8.80) \\
\end{array}$ & $\begin{array}{c}24.54 \\
(19.75)^{*}\end{array}$ & $\begin{array}{c}8.82 \\
(12.76)\end{array}$ & $\begin{array}{c}53.38 \\
(7.74)^{* *}\end{array}$ & $\begin{array}{c}44.35 \\
(11.74)\end{array}$ \\
\hline Acid Reflux/ GERD & $43(27.7)$ & $\begin{array}{l}35.31 \\
(7.92) \\
\end{array}$ & $\begin{array}{c}35.12 \\
(9.22) \\
\end{array}$ & $\begin{array}{c}15.12 \\
(16.83)^{*}\end{array}$ & $\begin{array}{c}8.23 \\
(12.45)\end{array}$ & $\begin{array}{c}48.56 \\
(10.56)^{*}\end{array}$ & $\begin{array}{c}43.79 \\
(11.91)\end{array}$ \\
\hline Chronic Sinusitis & $17(11)$ & $\begin{array}{l}36.01 \\
(7.54)\end{array}$ & $\begin{array}{l}35.07 \\
(9.02)\end{array}$ & $\begin{array}{c}10.41 \\
(13.96)\end{array}$ & $\begin{array}{c}10.11 \\
(14.16)\end{array}$ & $\begin{array}{c}47.29 \\
(14.18)\end{array}$ & $\begin{array}{c}44.84 \\
(11.79)\end{array}$ \\
\hline PCOS & $6(3.9)$ & $\begin{array}{l}41.23 \\
(9.14)\end{array}$ & $\begin{array}{l}34.93 \\
(8.79)\end{array}$ & $\begin{array}{c}14.83 \\
(15.55)\end{array}$ & $\begin{array}{c}9.95 \\
(14.05)\end{array}$ & $\begin{array}{l}40.83 \\
(8.73)\end{array}$ & $\begin{array}{c}45.28 \\
(11.81)\end{array}$ \\
\hline Migraines & $\begin{array}{c}23 \\
(14.8)\end{array}$ & $\begin{array}{l}36.91 \\
(9.63)\end{array}$ & $\begin{array}{l}34.87 \\
(8.72)\end{array}$ & $\begin{array}{c}10.52 \\
(16.06)\end{array}$ & $\begin{array}{c}10.08 \\
(13.79)\end{array}$ & $\begin{array}{c}40.57 \\
(11.07)^{*}\end{array}$ & $\begin{array}{c}45.90 \\
(11.68)\end{array}$ \\
\hline
\end{tabular}

*Difference between group with and without condition significant at $\mathrm{p}<.05$
${ }^{*}$ Difference between group with and without condition significant at $\mathrm{p}<.01$
Conditions not included in this table due to $\mathrm{n}<5$ include $\mathrm{CAD}$, heart disease other than CAD, heart attack, stroke, COPD,
Crohn's disease, celiac, chronic kidney disease, osteoporosis, fibromyalgia and chronic fatigue syndrome.

Participants reporting at least one musculoskeletal condition scored 4.45 points higher on average on the EFAS than those participants reporting that they did not have any of the musculoskeletal conditions $(P=.001$; table 3$)$. Individuals who reported at least one musculoskeletal condition had significantly higher BMIs than those who did not report any musculoskeletal conditions $(P=.014$; table 2). They were also significantly older $(P<.001)$ and reported more pain disability $(P<.001)$ than those without a musculoskeletal condition. Within the specific musculoskeletal conditions, participants who reported arthritis scored
6.07 points higher on average on the total EFAS than those who did not report arthritis $(P<.001)$. Participants with degenerative joint disease scored 6.81 points higher on average $(P<.001)$, and those reporting joint pain other than arthritis scored 4.33 points higher on average $(P=.006)$ compared to those who did not report these conditions (table 3). For each of these conditions, differences on the weight-specific subscale were greater than differences on either the musculoskeletal or cardio-respiratory subscales.

Table 3: EFAS Scores Based on Medical Conditions

\begin{tabular}{|c|c|c|c|c|c|c|c|c|c|}
\hline \multirow[t]{3}{*}{ Condition } & \multirow[t]{3}{*}{$\mathrm{n}(\%)$} & \multicolumn{2}{|c|}{$\begin{array}{c}\text { Total EFAS Score } \\
\text { Mean (SD) }\end{array}$} & \multicolumn{2}{|c|}{$\begin{array}{l}\text { EFAS Weight Specific } \\
\text { Fear Subscale } \\
\text { Mean (SD) }\end{array}$} & \multicolumn{2}{|c|}{$\begin{array}{c}\text { EFAS } \\
\text { Joint-Specific Fear } \\
\text { Subscale } \\
\text { Mean (SD) }\end{array}$} & \multicolumn{2}{|c|}{$\begin{array}{c}\text { EFAS } \\
\text { Cardiorespiratory } \\
\text { Fear Subscale } \\
\text { Mean (SD) }\end{array}$} \\
\hline & & & & & \multicolumn{3}{|c|}{ Condition Reported } & & \\
\hline & & Yes & No & Yes & No & Yes & No & Yes & No \\
\hline $\begin{array}{l}\text { Any } \\
\text { cardiorespirat } \\
\text { ory condition }\end{array}$ & $\begin{array}{c}109 \\
(70.3)\end{array}$ & $\begin{array}{c}34.78 \\
(7.96)^{* *}\end{array}$ & $\begin{array}{l}27.44 \\
(7.76)\end{array}$ & $\begin{array}{c}14.13 \\
(5.46)^{* *}\end{array}$ & $\begin{array}{l}10.05 \\
(4.30)\end{array}$ & $\begin{array}{c}12.96 \\
(2.93)^{* *}\end{array}$ & $\begin{array}{l}11.31 \\
(3.42)\end{array}$ & $\begin{array}{c}7.91 \\
(2.83)^{* *}\end{array}$ & $\begin{array}{c}6.08 \\
(1.78)\end{array}$ \\
\hline Hypertension & $\begin{array}{c}64 \\
(41.3)\end{array}$ & $\begin{array}{c}35.69 \\
(8.01)^{* *}\end{array}$ & $\begin{array}{l}30.96 \\
(8.35)\end{array}$ & $\begin{array}{c}14.53 \\
(5.54)^{* *}\end{array}$ & $\begin{array}{l}12.10 \\
(5.23)\end{array}$ & $\begin{array}{c}13.16 \\
(2.85)^{*}\end{array}$ & $\begin{array}{l}12.11 \\
(3.27)\end{array}$ & $\begin{array}{c}8.00 \\
(2.62)^{*}\end{array}$ & $\begin{array}{c}7.05 \\
(2.74)\end{array}$ \\
\hline
\end{tabular}




\begin{tabular}{|c|c|c|c|c|c|c|c|c|c|}
\hline $\begin{array}{l}\text { Mitral valve } \\
\text { prolapse }\end{array}$ & $14(9)$ & $\begin{array}{l}34.21 \\
(7.26)\end{array}$ & $\begin{array}{l}32.79 \\
(8.64)\end{array}$ & $\begin{array}{l}13.92 \\
(5.73)\end{array}$ & $\begin{array}{l}13.02 \\
(5.47)\end{array}$ & $\begin{array}{l}12.79 \\
(2.36)\end{array}$ & $\begin{array}{l}12.52 \\
(3.21)\end{array}$ & $7.5(2.65)$ & $\begin{array}{c}7.44 \\
(2.74)\end{array}$ \\
\hline Heart murmur & $10(6.5)$ & $\begin{array}{c}36.80 \\
(4.66)^{*}\end{array}$ & $\begin{array}{l}32.65 \\
(8.66) \\
\end{array}$ & $\begin{array}{c}16.80 \\
(3.52)^{* *}\end{array}$ & $\begin{array}{l}12.85 \\
(5.50) \\
\end{array}$ & $\begin{array}{l}12.50 \\
(2.12) \\
\end{array}$ & $\begin{array}{l}12.55 \\
(3.20) \\
\end{array}$ & $\begin{array}{c}7.50 \\
(2.55) \\
\end{array}$ & $\begin{array}{c}7.44 \\
(2.74) \\
\end{array}$ \\
\hline $\begin{array}{l}\text { Pulmonary } \\
\text { hypertension }\end{array}$ & $5(3.2)$ & $\begin{array}{c}40.60 \\
(6.23)^{*}\end{array}$ & $\begin{array}{l}32.66 \\
(8.47)\end{array}$ & $\begin{array}{l}16.00 \\
(6.16)\end{array}$ & $\begin{array}{l}13.01 \\
(5.45)\end{array}$ & $\begin{array}{l}14.40 \\
(3.91)\end{array}$ & $\begin{array}{l}12.48 \\
(3.10)\end{array}$ & $\begin{array}{c}10.20 \\
(3.11)^{*}\end{array}$ & $\begin{array}{c}7.35 \\
(2.67)\end{array}$ \\
\hline Asthma & $\begin{array}{c}24 \\
(15.5) \\
\end{array}$ & $\begin{array}{r}34.38 \\
(9.15) \\
\end{array}$ & $\begin{array}{l}32.65 \\
(8.40) \\
\end{array}$ & $\begin{array}{l}13.42 \\
(6.06)\end{array}$ & $\begin{array}{l}13.05 \\
(5.39) \\
\end{array}$ & $\begin{array}{c}13.13 \\
(12.44) \\
\end{array}$ & $\begin{array}{l}12.44 \\
(3.16) \\
\end{array}$ & $\begin{array}{c}7.83 \\
(2.73)\end{array}$ & $\begin{array}{c}7.37 \\
(2.73)\end{array}$ \\
\hline Diabetes & $\begin{array}{c}23 \\
(14.8)\end{array}$ & $\begin{array}{l}35.48 \\
(8.04)\end{array}$ & $\begin{array}{l}32.47 \\
(8.54) \\
\end{array}$ & $\begin{array}{l}13.78 \\
(4.99)\end{array}$ & $\begin{array}{l}12.98 \\
(5.57) \\
\end{array}$ & $\begin{array}{l}13.35 \\
(3.46)\end{array}$ & $\begin{array}{l}12.40 \\
(3.07)\end{array}$ & $\begin{array}{c}8.35 \\
(2.81)\end{array}$ & $\begin{array}{l}2.69 \\
(.23)\end{array}$ \\
\hline $\begin{array}{l}\text { High } \\
\text { Cholesterol }\end{array}$ & $\begin{array}{c}60 \\
(38.7) \\
\end{array}$ & $\begin{array}{c}35.58 \\
(7.88)^{* *}\end{array}$ & $\begin{array}{l}31.22 \\
(8.50) \\
\end{array}$ & $\begin{array}{c}14.53 \\
(5.18)^{* *}\end{array}$ & $\begin{array}{l}12.20 \\
(5.49)\end{array}$ & $\begin{array}{l}13.00 \\
(2.88) \\
\end{array}$ & $\begin{array}{l}12.26 \\
(3.27) \\
\end{array}$ & $\begin{array}{c}8.05 \\
(2.70)^{*}\end{array}$ & $\begin{array}{c}7.06 \\
(2.68) \\
\end{array}$ \\
\hline Sleep Apnea & $\begin{array}{c}43 \\
(27.7) \\
\end{array}$ & $\begin{array}{c}36.57 \\
(7.40)^{* *}\end{array}$ & $\begin{array}{l}31.55 \\
(8.53) \\
\end{array}$ & $\begin{array}{c}15.23 \\
(5.27)^{* *}\end{array}$ & $\begin{array}{l}12.29 \\
(5.36) \\
\end{array}$ & $\begin{array}{l}13.29 \\
(2.76) \\
\end{array}$ & $\begin{array}{l}12.27 \\
(3.23) \\
\end{array}$ & $\begin{array}{c}8.60 \\
(3.16)^{* *} \\
\end{array}$ & $\begin{array}{c}7.00 \\
(2.41) \\
\end{array}$ \\
\hline $\begin{array}{l}\text { Any } \\
\text { musculoskelet } \\
\text { al condition }\end{array}$ & $\begin{array}{c}70 \\
(45.2)\end{array}$ & $\begin{array}{c}35.38 \\
(7.70)^{* *}\end{array}$ & $\begin{array}{l}30.93 \\
(8.66)\end{array}$ & $\begin{array}{c}14.60 \\
(5.40)^{* *}\end{array}$ & $\begin{array}{l}11.87 \\
(5.26)\end{array}$ & $\begin{array}{c}13.38 \\
(2.96)^{* *}\end{array}$ & $\begin{array}{l}11.87 \\
(5.26)\end{array}$ & $\begin{array}{c}7.76 \\
(3.00)\end{array}$ & $\begin{array}{c}7.19 \\
(2.46)\end{array}$ \\
\hline Arthritis & $\begin{array}{c}42 \\
(27.1) \\
\end{array}$ & $\begin{array}{c}37.33 \\
(7.48)^{* *}\end{array}$ & $\begin{array}{c}31.2 \\
(8.31)\end{array}$ & $\begin{array}{c}15.40 \\
(5.37)^{* *}\end{array}$ & $\begin{array}{l}12.25 \\
(5.29) \\
\end{array}$ & $\begin{array}{c}13.79 \\
(2.79)^{* *}\end{array}$ & $\begin{array}{l}12.08 \\
(3.14) \\
\end{array}$ & $\begin{array}{c}8.14 \\
(2.72) \\
\end{array}$ & $\begin{array}{c}7.19 \\
(2.69) \\
\end{array}$ \\
\hline $\begin{array}{l}\text { Joint pain } \\
\text { other than } \\
\text { arthritis }\end{array}$ & $\begin{array}{c}24 \\
(15.5)\end{array}$ & $\begin{array}{c}36.61 \\
(6.11)^{* *}\end{array}$ & $\begin{array}{l}32.27 \\
(8.73)\end{array}$ & $\begin{array}{c}15.38 \\
(5.08)^{*}\end{array}$ & $\begin{array}{l}12.69 \\
(5.46)\end{array}$ & $\begin{array}{c}13.78 \\
(2.78)^{*}\end{array}$ & $\begin{array}{l}12.33 \\
(3.15)\end{array}$ & $\begin{array}{c}8.46 \\
(3.44)^{*}\end{array}$ & $\begin{array}{c}7.26 \\
(2.54)\end{array}$ \\
\hline $\begin{array}{l}\text { Plantar } \\
\text { fasciitis }\end{array}$ & $\begin{array}{c}29 \\
(18.7)\end{array}$ & $\begin{array}{l}35.17 \\
(7.75) \\
\end{array}$ & $\begin{array}{l}32.40 \\
(8.62) \\
\end{array}$ & $\begin{array}{l}14.59 \\
(5.12)\end{array}$ & $\begin{array}{l}12.76 \\
(5.52) \\
\end{array}$ & $\begin{array}{l}13.55 \\
(3.15) \\
\end{array}$ & $\begin{array}{l}12.31 \\
(3.10)\end{array}$ & $\begin{array}{c}7.03 \\
(2.21) \\
\end{array}$ & $\begin{array}{c}7.54 \\
(2.83) \\
\end{array}$ \\
\hline Herniated Disc & $17(11)$ & $\begin{array}{l}36.24 \\
(7.00) \\
\end{array}$ & $\begin{array}{l}32.51 \\
(8.62) \\
\end{array}$ & $\begin{array}{l}15.41 \\
(5.23)\end{array}$ & $\begin{array}{l}12.82 \\
(5.46) \\
\end{array}$ & $\begin{array}{l}12.59 \\
(1.80) \\
\end{array}$ & $\begin{array}{l}12.54 \\
(3.27)\end{array}$ & $\begin{array}{c}8.24 \\
(2.68) \\
\end{array}$ & $\begin{array}{c}7.35 \\
(2.72) \\
\end{array}$ \\
\hline $\begin{array}{l}\text { Degenerative } \\
\text { Joint Disease }\end{array}$ & $13(8.4)$ & $\begin{array}{c}39.15 \\
(6.18)^{* *} \\
\end{array}$ & $\begin{array}{r}32.35 \\
(8.48) \\
\end{array}$ & $\begin{array}{c}16.85 \\
(4.63)^{* *}\end{array}$ & $\begin{array}{l}12.76 \\
(5.43) \\
\end{array}$ & $\begin{array}{l}13.54 \\
(1.76) \\
\end{array}$ & $\begin{array}{l}12.45 \\
(3.22) \\
\end{array}$ & $\begin{array}{c}8.77 \\
(2.59) \\
\end{array}$ & $\begin{array}{c}7.32 \\
(2.71) \\
\end{array}$ \\
\hline $\begin{array}{l}\text { Acid Reflux/ } \\
\text { GERD }\end{array}$ & $43(27.7)$ & $\begin{array}{l}34.53 \\
(7.47) \\
\end{array}$ & $\begin{array}{l}32.30 \\
(8.84) \\
\end{array}$ & $\begin{array}{l}13.60 \\
(4.84) \\
\end{array}$ & $\begin{array}{l}12.91 \\
(5.71) \\
\end{array}$ & $\begin{array}{c}13.35 \\
(3.07)^{*}\end{array}$ & $\begin{array}{l}12.23 \\
(3.12) \\
\end{array}$ & $\begin{array}{c}7.58 \\
(2.44) \\
\end{array}$ & $\begin{array}{c}7.39 \\
(2.83) \\
\end{array}$ \\
\hline $\begin{array}{l}\text { Chronic } \\
\text { Sinusitis }\end{array}$ & $17(11)$ & $\begin{array}{l}34.12 \\
(7.38)\end{array}$ & $\begin{array}{l}32.77 \\
(8.66) \\
\end{array}$ & $\begin{array}{l}13.35 \\
(4.27) \\
\end{array}$ & $\begin{array}{l}13.07 \\
(5.62)\end{array}$ & $\begin{array}{l}13.71) \\
(3.72)\end{array}$ & $\begin{array}{l}12.40 \\
(3.04)\end{array}$ & $\begin{array}{c}7.06 \\
(2.25) \\
\end{array}$ & $\begin{array}{c}7.49 \\
(2.78)\end{array}$ \\
\hline PCOS & $6(3.9)$ & $\begin{array}{l}34.83 \\
(5.71) \\
\end{array}$ & $\begin{array}{l}32.84 \\
(8.61) \\
\end{array}$ & $\begin{array}{l}14.16 \\
(6.55) \\
\end{array}$ & $\begin{array}{l}13.06 \\
(5.45) \\
\end{array}$ & $\begin{array}{l}13.50 \\
(3.02) \\
\end{array}$ & $\begin{array}{l}12.51 \\
(3.14) \\
\end{array}$ & $\begin{array}{c}7.17 \\
(2.32) \\
\end{array}$ & $\begin{array}{c}7.46 \\
(2.74) \\
\end{array}$ \\
\hline Migraines & $\begin{array}{c}23 \\
(14.8)\end{array}$ & $31.91(9.08)$ & $\begin{array}{c}33.10 \\
(8.43)\end{array}$ & $\begin{array}{c}13.17 \\
(13.09)\end{array}$ & $\begin{array}{l}13.09 \\
(5.35)\end{array}$ & $\begin{array}{l}11.56 \\
(2.63)\end{array}$ & $\begin{array}{l}12.72 \\
(3.19)\end{array}$ & $\begin{array}{c}7.17 \\
(2.35)\end{array}$ & $\begin{array}{c}7.49 \\
(2.79)\end{array}$ \\
\hline
\end{tabular}

${ }^{*}$ Difference between group with and without condition significant at $\mathrm{p}<.05$

** Difference between group with and without condition significant at $\mathrm{p}<.01$

Conditions not included in this table due to $\mathrm{n}<5$ include CAD, heart disease other than CAD, heart attack, stroke, COPD,

Crohn's disease, celiac, chronic kidney disease, osteoporosis, fibromyalgia and chronic fatigue syndrome.

The initial regression model included BMI, PDI and age. Each of these three predictors contributed significantly to the model (BMI $\beta=.22, P=.003$; PDI $\beta=.27, P<.001$; age $\beta=.25$, $P=.001)$. This model accounted for $28.7 \%$ $(P<.001)$ of variance in total EFAS scale scores. Medical conditions that demonstrated a significant difference in EFAS between groups that reported and did not report the condition were included in the regression analyses. Each condition was added separately to create a new model. None of the medical conditions contributed significantly to the initial model (table 4). 
Review of the collinearity statistics suggested that multicollinearity did not account for the non-significance results in addition of medical condition to the regression analyses. Correlations between each of the initial predictors (BMI, pain and age) and each medical condition were low, and collinearity statistics suggested multicollinearity was not present in these analyses (VIF range 1.041.47).

Table 4: Amount of Variance in Total EFAS Scores Explained by Medical Conditions

\begin{tabular}{|c|c|c|c|c|c|}
\hline Model & Variables Entered/Added & $\mathrm{R}^{2}$ & $\mathrm{R}^{2}$ Change & Model Significance & Significance of $\mathrm{R}^{2}$ Change \\
\hline $\begin{array}{c}\text { Initial } \\
\text { Model }\end{array}$ & $\begin{array}{c}\text { BMI } \\
\text { PDI }\end{array}$ & .287 & & \\
& Age & & & \\
\hline 1 & Any cardiorespiratory condition & .301 & .013 & & .094 \\
\hline 2 & Hypertension & .290 & .003 & .431 \\
\hline 3 & Heart Murmur & .288 & .001 & & .748 \\
\hline 4 & Pulmonary Hypertension & .290 & .003 & .439 & .143 \\
\hline 5 & High Cholesterol & .297 & .010 & & .185 \\
\hline 6 & Obstructive Sleep Apnea & .296 & .008 & & .780 \\
\hline 7 & Any musculoskeletal condition & .288 & .000 & & .596 \\
\hline 8 & Arthritis & .289 & .001 & & .685 \\
\hline 9 & Joint Pain (not arthritis) & .288 & .001 & & .386 \\
\hline 10 & Degenerative Joint Disease & .291 & .004 & & \\
\hline
\end{tabular}

\section{Discussion}

This study assessed the differences in exercise fear avoidance beliefs in adults who had various chronic medical conditions. Specifically, we examined the differences between exercise fear avoidance beliefs in individuals who reported musculoskeletal conditions and cardiorespiratory conditions and risk factors. Individuals who reported having at least one condition scored significantly higher on the EFAS than those individuals who reported not having any of the conditions. Specific conditions that were associated with higher fear avoidance scores were hypertension, heart murmur, pulmonary hypertension, high cholesterol, obstructive sleep apnea, arthritis, degenerative joint disease, or joint pain other than arthritis.

Despite having significantly higher EFAS scores, the presence of a medical condition did not explain a significant amount of variance in EFAS scores. This could be due, in part, to the differences in other characteristics between those individuals with and without medical conditions. Specifically, BMI was significantly higher among individuals reporting at least one condition. Within the individual conditions, those individuals with hypertension, obstructive sleep apnea, arthritis and joint pain other than arthritis all had significantly higher BMIs than individuals who did not report these conditions. These groups also reported significantly higher levels of pain disability, and they were significantly older than those not reporting the conditions. BMI, pain and age all significantly contributed to the initial model, indicating that these characteristics may be more important in the development of exercise fear avoidance beliefs than the presence of a musculoskeletal or cardiorespiratory condition.

There are limitations to this study which should be noted. This study was conducted with generally healthy adults (not currently hospitalized for acute illness or under instructions to avoid exercise due to uncontrolled medical conditions), and all medical conditions were self-reported. The 
fear-avoidance model proposes that activity avoidance is predictive as well as responsive. Fear may begin as a direct response to discomfort experienced after a movement; however, if an individual believes that the discomfort is the signal of physical harm, he may begin to avoid any activity that he believes will result in similar discomfort (Lethem, et al., 1983; Kori, Miller, \& Todd, 1990). We hypothesized that individuals who believe they have a specific condition may similarly avoid activity due to the perceived belief that their risk of harm is increased by the presence of this condition. For this reason, we wanted to capture participants' self-reported conditions. There were significant differences between these groups based on self-report, however future studies should continue this line of exploration by assessing the differences between individuals with documented diagnoses and self-reported conditions. Additionally, we asked participants to report if they had ever been told they had these conditions. The reason for asking this question in this way was to capture all individuals who had been told they had a condition, rather than only those actively being treated for a condition. Future research should explore differences in individuals with active diagnoses compared to those with resolved conditions to explore if fear levels are associated with the present status of a condition.

Exercise fear-avoidance may be a significant barrier to adopting a regular physical activity routine, especially among adults who are overweight or obese or who suffer from higher levels of pain (Elfving, Andersson \& Grooten, 2007; Wingo et al., 2011, Wingo et al., 2013). Understanding patient characteristics that are likely to lead to the development of fear avoidance beliefs will allow for more efficient screening of these beliefs, as well as a better understanding of how to tailor exercise prescriptions to meet the needs of individual with these fears. While individuals with musculoskeletal or cardiorespiratory conditions may have higher fear-avoidance beliefs than those without these conditions, it appears that BMI, pain and age are three of the key patient characteristics associated with exercise fearavoidance beliefs.

\section{References}

Ekkekakis, P. \& Lind, E. (2006). "Exercise does not feel the Same When You are Overweight: The Impact of Self-Selected and Imposed Intensity on Affect and Exertion," International Journal of Obesity, 30: 652-660.

Elfving, B. T., Andersson, T. \& Grooten, W. (2007). "Low Levels of Physical Activity in Back Pain Patients are Associated with High Levels of Fear-Avoidance Beliefs and Pain Catastrophizing," Physiotherapy Research International, 12 (1), 14-24.

Garrow, J. S. \& Webster, J. (1985). "Quetelet's Index (W/H2) as a Measure of Fatness," International Journal of Obesity, 9:147-153.

Kori, S. H., Miller, R. P. \& Todd, D. D. (1990). 'Kinesiophobia: A New View of Chronic Pain Behavior,' Pain Management, Jan/Feb. 35-43.

Lethem, J., Slade, P. D., Troup, J. D. G. \& Bentley, G. (1983). "Outline of a FearAvoidance Model of Exaggerated Pain Perceptions," Behavior Research and Therapy, 21:401-408.

Nevitt, M. C. \& Lane, N. (1999). "Body Weight and Osteoarthritis," American Journal of Medicine, 107: 632-633.

Pollard, C. A. (1984). "Preliminary Validity Study of the Pain Disability Index," Perceptual and Motor Skills, 59, 974.

Tukker, A., Visscher, T. L. \& Picavet. H. S. J. (2009). "Overweight and Health Problems of the Lower Extremities: Osteoarthritis, Pain and Disability," Public Health Nutrition,12 (3): 359-368.

Vlaeyen, J. W. S., Kole-Snijders, A. M. J., Rotteveel, A. M., et al. (1995). "The Role of Fear of Movement/(re)Injury in Pain 
Disability," Journal of Occupational Rehabilitation, 5: 235-252.

Wingo, B. C., Baskin, M., Ard, J. D., et al. (2013). "Component Analysis and Initial Validity of the Exercise Fear Avoidance Scale," American Journal of Health Behavior, 37(1): 87-95.

Wingo, B. C., Evans, R. R., Ard, J. D., et al. (2011). "Fear of Physical Response to Exercise among Overweight and Obese Adults," Qualitative Research in Sport and Exercise, 3(2): 174-192. 